\title{
XACML Policies for Exclusive Resource Usage
}

\author{
Vijayant Dhankhar, Saket Kaushik, and Duminda Wijesekera \\ Department of Information \& Software Engineering \\ George Mason University \\ Fairfax, VA 22030, U.S.A \\ \{vohankha, skaushik, dwijesek\}@gmu.edu
}

\begin{abstract}
The extensible access control markup language (XACML) is the standard access control policy specification language of the World Wide Web. XACML does not provide exclusive accesses to globally resources. We do so by enhancing the policy execution framework with locks.
\end{abstract}

\section{Introduction}

The extensible access control markup language (XACML) [18] is the standard language to specify accesses to resources available on the world wide web. However, the XACML normative specifications lack necessary syntax to specify exclusive access to resources, and furthermore, publicly available XACML policy enforcement frameworks do not enforce them. Given that web orchestrations are composed from existing ones using languages such as BPEL [16] may pose concurrent request for exclusively usable resources (such as updating an XML schema), we enhance XACML syntax and enforcement mechanisms to do so.

Perils of not using a synchronization mechanism (such as the dirty read [21] in distributed systems) in exclusive accesses are well known. Consequently, we advocate to make a distinction in granting exclusive access and non-exclusive accesses by access controllers. Thus, we add appropriate syntax to XACML and an enforcement mechanism using locks. Consequently, if and when granted, the access control policy is aware that such permissions are granted in exclusion. This enrichment to XACML has no relationship to application level concurrency control, but not surprisingly, due to the enforced semantic distinction between exclusive and non exclusive acccesses, aids in enforcing separation of duty principles [12|8|9|20].

To enforce enhanced XACML policies, we add a lock manager to the policy enforcement module and require that all globally accessible resources register with a unique lock manager. In order to ensure starvation avoidance, we assume that resource requesters give up such resource after their usage - although this latter aspect is being driven by policy in our ongoing work.

The rest of the paper is organized as follows. Section 2 has related work. Section 3 presents sample Use Cases and Misuse Cases of exclusive access and our design to realize the former. Syntactic extensions to XACML appear in Section 4 and Section 5 describes the architectural enhancements used to enforce locking. Section 6 describes our implementation and Section 7 informally argues that our locking ensures safety and liveliness. Finally, Section 8 concludes the paper. 


\section{Related Work}

Motivated by a desire to to introduce trust-based, context-aware access control framework for Web Service invocations, including support for RBAC sessions, Bhatti et al., 46517] define X-RBAC and X-GTRBAC models for access control frameworks for Web Services, respectively based on RBAC [12] and GTRBAC [14] models of access control. However, they do not provide mechanisms to enforce dynamic separation of duty (DSoD) policies, as is the case with current XACML RBAC profile [17]. Cardea by Lepro et al. [15] offers a dynamic access control system for the Web, where the $d y$ namism means that the request is not bound to local identities at runtime, but instead uses a remote requester's context instead. However, Cardea does not explicitly address concurrent access to exclusively used resources nor dynamic separation of duty policies.

\section{Use Cases, Misuse Cases and Requirements}

Although some existing work on Web Services orchestration argues the need to lock shared resources [3[13], to the best of our knowledge they only reserving syntax for locks [3]. Following Use Cases show the need.

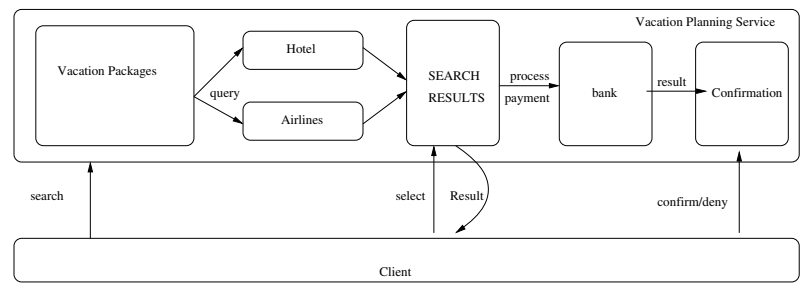

Fig. 1. Vacation Planning Service

\subsection{Use Case 1: Exclusive Access}

Consider an example, vacation planning service (VPlanner) that reserves hotel rooms and air tickets for its clients, whose work-flow is given in figure1][23]. As seen, this interactive service is used to first searches for available rooms and air tickets for specified dates and destinations and presents various alternatives to its clients, from which the latter chooses alternative for reservations. The service then initiates a monetary transfer request to the credit granting agency. On success, the room(s) and air tickets are reserved and aborted otherwise. An efficient implementation should invoke airline and hotel room searches concurrently, while, work-flow dependencies require that monetary transfer request should wait till other parts of the procedure are complete.

Now, suppose two clients are searching for reservations from the VPlanner and are shown the same tickets and hotel rooms. This is potentially dangerous because both can choose the same room or ticket, where simultaneous requests can deadlock two BPEL server processes. One way of avoiding this situation is to not offer a second 
client the choices while a precedent client $s$ in the process of reserving a package - thus requiring the VPlanner to lock rooms and tickets during ongoing reservations, referred to as tentative locking of resources in the Web Services literature [3].

\subsection{Use Case 2: Enforcing Dynamic Constraints}

Example 1 (DSoD [8]). Consider a DSoD constraint an employee cannot invoke role 1 in a session if another role, role 2, is already invoked in some other session. Assuming a data structure maintained by the system 'sessions' with following XML schema:

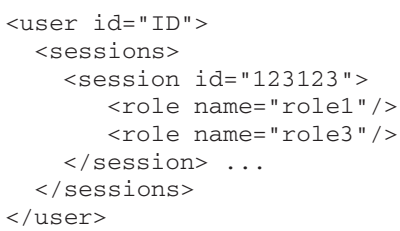

An abbreviated DSoD XACML policy as follows:

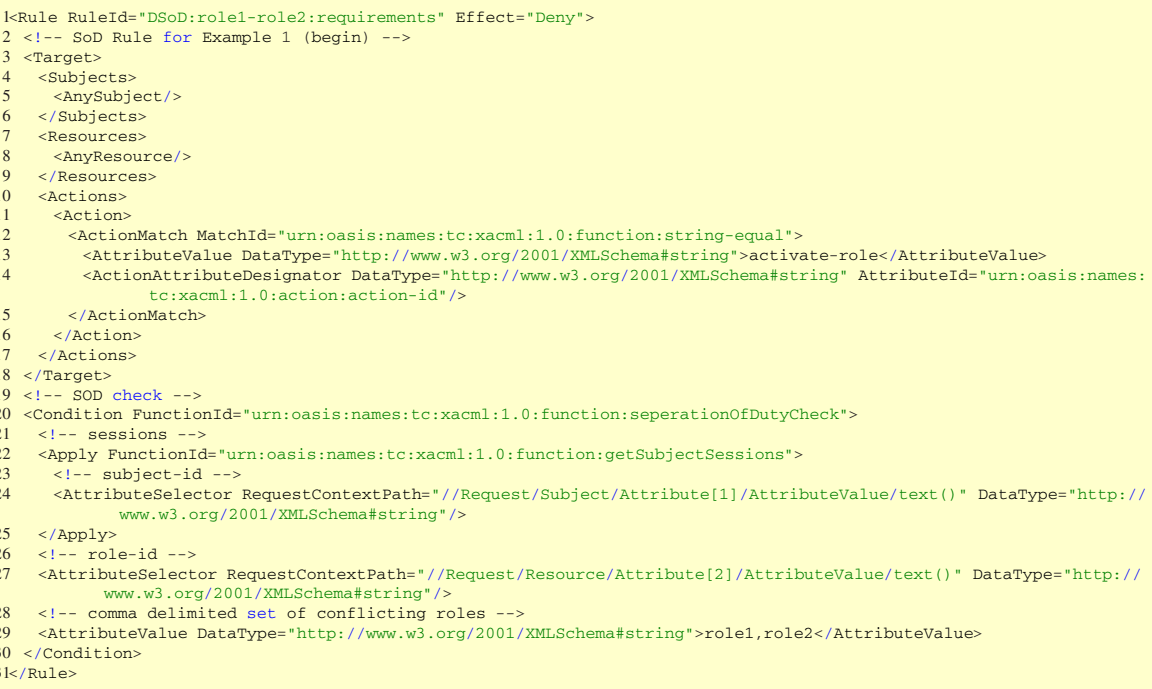

Policy 1. DSoD policy

Example 1 above is a DSoD policy expressed in terms of the XACML RBAC profile [17], where as stated in lines 20-30, role 1 and role 2 cannot be co-activated. However, this policy is not currently enforceable because XACML enforcement does not consider concurrent requests. To be fair, the XACML RBAC profile outsources the process of enabling roles to the Role Enablement Authority module.

Our design enables the follwing Use cases:

Secure registration of resources: A resource may register itself to a unique lock manager.

Secure deregistration of resources: Only a resource is able to securely deregister itself from the lock manager. 


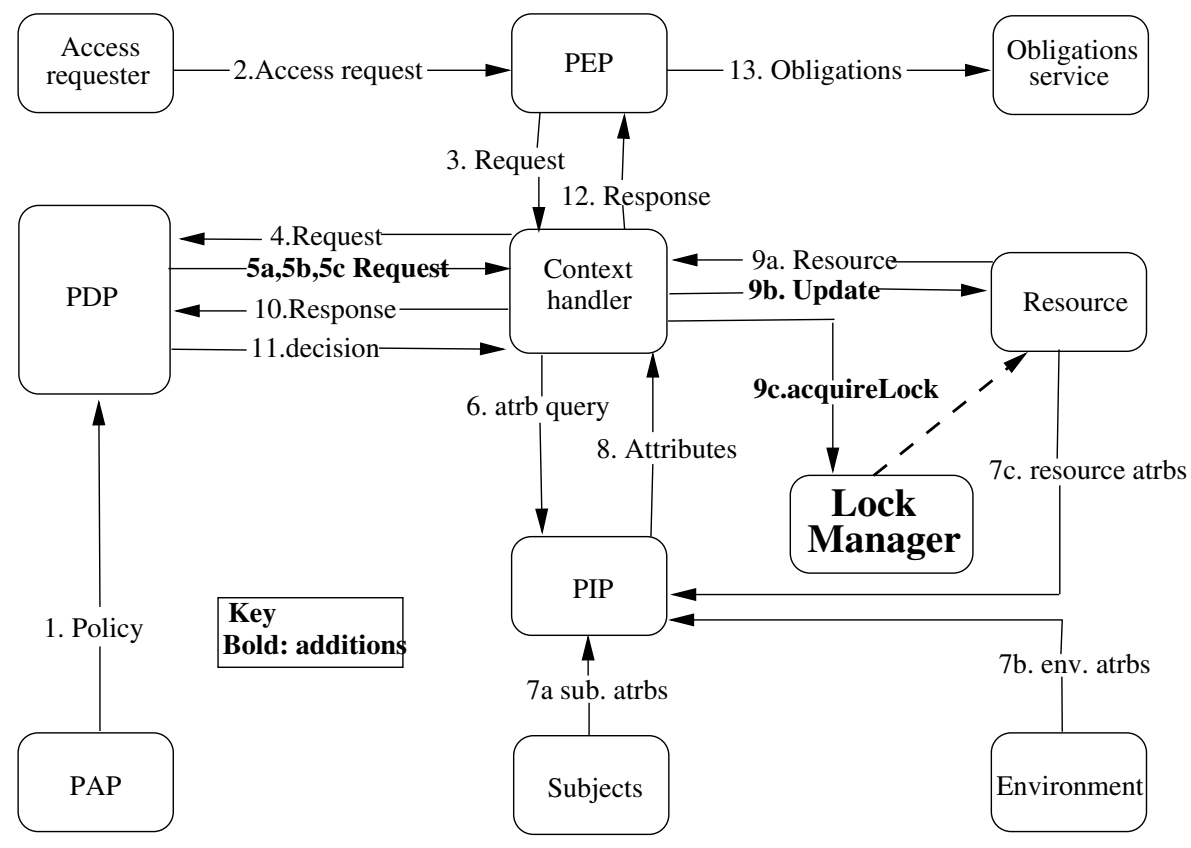

Fig. 2. Extended XACML data flow diagram

Exclusive access /relinquish resources: Exclusive use of a resource must be granted to a unique requester at any given time.

\subsection{Preventing Misuse Cases}

Our design prevents following Misuse Cases:

Registering a resource with multiple lock managers: An exclusively usable resource being registered with multiple lock managers, referred to as singular registration.

Spoofing a resource: Others (de)registering an exclusively accessible resource.

Preventing simultaneous exclusive access: Multiple requesters simultaneous accessing an exclusively usable resource.

Starvation: Refusing exclusive access to resources when not in use.

\section{Enhancing the XACML Syntax}

Because our solution use locks, we add them to XACML syntax. Each of the following elements are specified within $<$ Rule/ $>$, $<$ Policy $/>$ and $<$ Policyset $/>$ elements of XACML.

- $<$ PreAction / $>$ specifies a set of locks to be acquired before rule evaluation.

$<$ AcquireLocks / > specifies a set of locks to be acquired and is a sub element of $<$ PreAction/> element, where the $<$ AcquireLock $>$ sub element specifies an individual lock. 
- <PostAction / > identifies a set of actions to be performed after (a) rule evaluation leads to a permitted request, (b) rule evaluation leads to a denied request. The set of actions may include releasing locks or updating system resources. For different evaluation results multiple $<$ Postaction / > elements may be defined.

Effect attribute indicate the effect of a post action, as discussed above.

$<$ Updates /> specifies updates to be performed in a < PostAction/>. <Update> sub element specifies an individual change.

$<$ ReleaseLocks / > specifies a set of locks to release and is a sub element of $<$ PostAction/> element. <ReleaseLock > sub element specifies an individual lock.

Introduced elements specify lock acquisition prerequisite for evaluating a rule and post evaluation steps to be taken. The following example policy extends policy 1 with proposed syntactic enhancements.

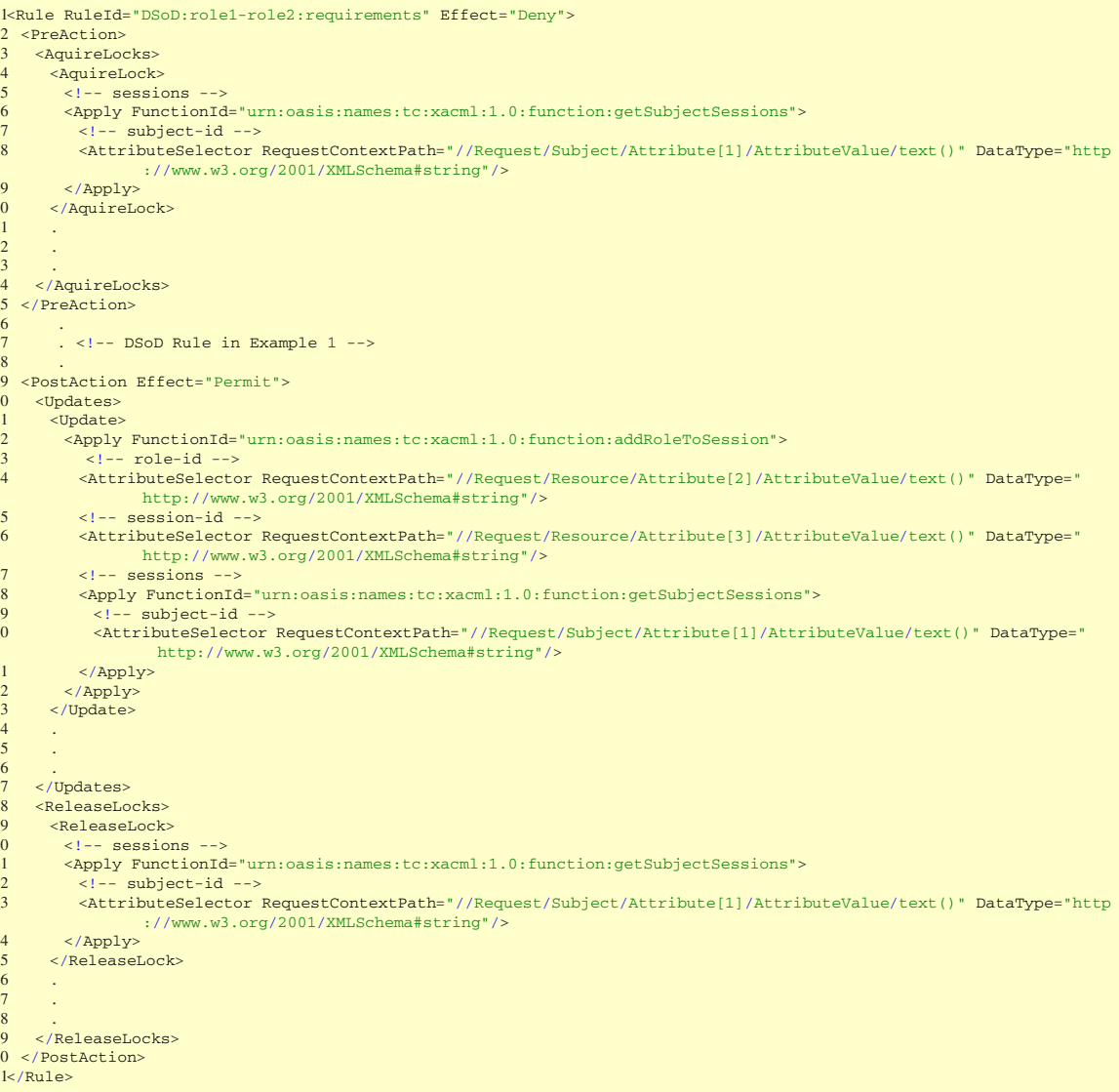

Policy 3. Enhancements to DSoD policy

The PreAction element in lines (2-15) of policy 3 above states that before evaluating a rule in the DSoD policy, the user session must be locked (lines 5-10). Similarly, 
PostAction element in lines (19-50) requires that after the policy has been evaluated, the locks acquired earlier be released for future concurrency-free changes to user sessions. We assume here that resources are not created during XACML evaluation (they already exist and are registered with a lock manager), however their usage status, i.e., open for read/write, etc., may be modified during a policy evaluation. For example, an XACML evaluation can modify a log file, etc.

\subsection{Implemented Semantics of Syntactic Extensions}

Postaction elements are evaluated in the following manner.

- Post action only updates resources for locks obtained at the corresponding level or those obtained at the level of the container.

- If two rules within a policy require same lock then they must be acquired and released at Policy level. Such locks are visible within all embedded rules. Similarly, if a lock is acquired at the Policyset level then it is visible to all embedded policies.

- If a resource must be updated in multiple rules, then corresponding lock must be acquired at their container level, i.e., Policy.

- Rule evaluation within a Policy element is evaluated by a single thread of execution.

- If locks are required at only the rule level, they must be released at the rule level $<$ PostAction/ $>$, otherwise, they must be released at the $<$ Policy/ $>$ level $<$ PostAction/ $>$

\subsection{XACML Functions}

Extensions to policy syntax is achieved with the help of following functions:

function:getSubjectSessions(\$subject-id as string). Accepts a subject-id as an input and returns a bag of session objects used by this subject. If the subject's sessions have been acquired by PDP through exclusive access, i.e., locked, then the sessions are cached till the lock to the sessions is released.

function:addRoleToSession(\$role-id as string, \$session-id as string, \$sessions as bag). Accepts role-id, session-id and a bag of sessions as input and it adds the passed role to the particular session. Sessions data structure contains all the sessions and session-id is used to locate the relevant session in the current implementation, although more efficient implementations are possible.

\section{Architectureral Enhancements Needed for Locks}

Figure 2 shows the existing XACML execution model with data flow for policy control [18]. The data flow begins with the Policy Administration Point (PAP) that authors the policies evaluated by the XACML framework, shown in Flow 1. Next, access requests (Flow 2), initiated by resource requesters, are intercepted by the Policy Enforcement Point (PEP). PEP forwards them, Flow 3, to the Context Handler (CH) with optional requester attributes and environmental conditions required for processing. Context handler has following three functions: 
Flow 4: Translate access requests into a format understood by the Policy Decision Point (PDP).

Flow 10: In response to Flow 5, generate the evaluation context by gathering resource, requester attributes and current system state of from the policy information point (PIP) (i.e. from, Flows 6,7,8 and 9), and pass them to the PDP.

Flow 12: Receive policy decisions from the PDP and translate them back to the PEP.

PDP evaluates an XACML policy applicable to the access request and accompanying context. If fails, the access is denied and granted otherwise. This decision is made available to the context handler of Flow 11 and is relayed to the PEP for enforcement.

Figure 2 shows the extended XACML data flow diagram that introduces a lock manager $(L M)$ to augment XACML access control decisions. The Lock Manager grants and revokes locks for accessing resources registered with itself, requiring extra data flows as follows:

Flow 5b - Update System Request (USR): May be initiated by the PDP to update system resources for setting up an access. For example, enabling a role may require that the user session (a system resource) be updated.

Flow 5c - Create Lock Request (CRL): Is initiated by the PDP on behalf of the requesting process, in response for exclusive access to an available resource. This is finally refined to the acquireLock operation (9c.), where the lock is owned by the requesting process.

Flow 10 - Response to PDP queries (overloaded): We reuse the response sent by the context handler to the PDP queries for sending USR and CRL responses in addition to resource query response.

Flow 9b - Resource update, 9b: Upon enforcing the access control decision, the PEP updates an internal log of accesses. This data flow enables enforcing history based resource usage.

Flow 9c - AcquireLock, 9c: Instructs the LM to invoke a lock on behalf of a requester. Based on the availability of a resource, this operation may succeed or fail.

As in the normative XACML specification, we assume that all attributes have been authenticated (using attribute certificate authenticity) prior to policy evaluation. We discuss the additional complexity due to these addition later in section 5.2 First, we describe the Lock Manager design.

\subsection{Lock Manager (LM)}

The Lock Manager (see figure 2) is a privileged process that, at any given time, has only a single instance running. The Lock Manager maintains and creates locks for resources it manages. The functionality of the Lock Manager that provides and maintains locks are as follows:

Lock Acquisition. Lock acquisition is an atomic operation acquireLock, implemented using an atomic operation, akin to the unix test\&set operation [22], with two associated strings - requester Id and resourceId with the acquireLock. These strings can be qualified names or URIs of network entities. The actual call is made by the lock manager within a critical section, as shown in section 6 . 


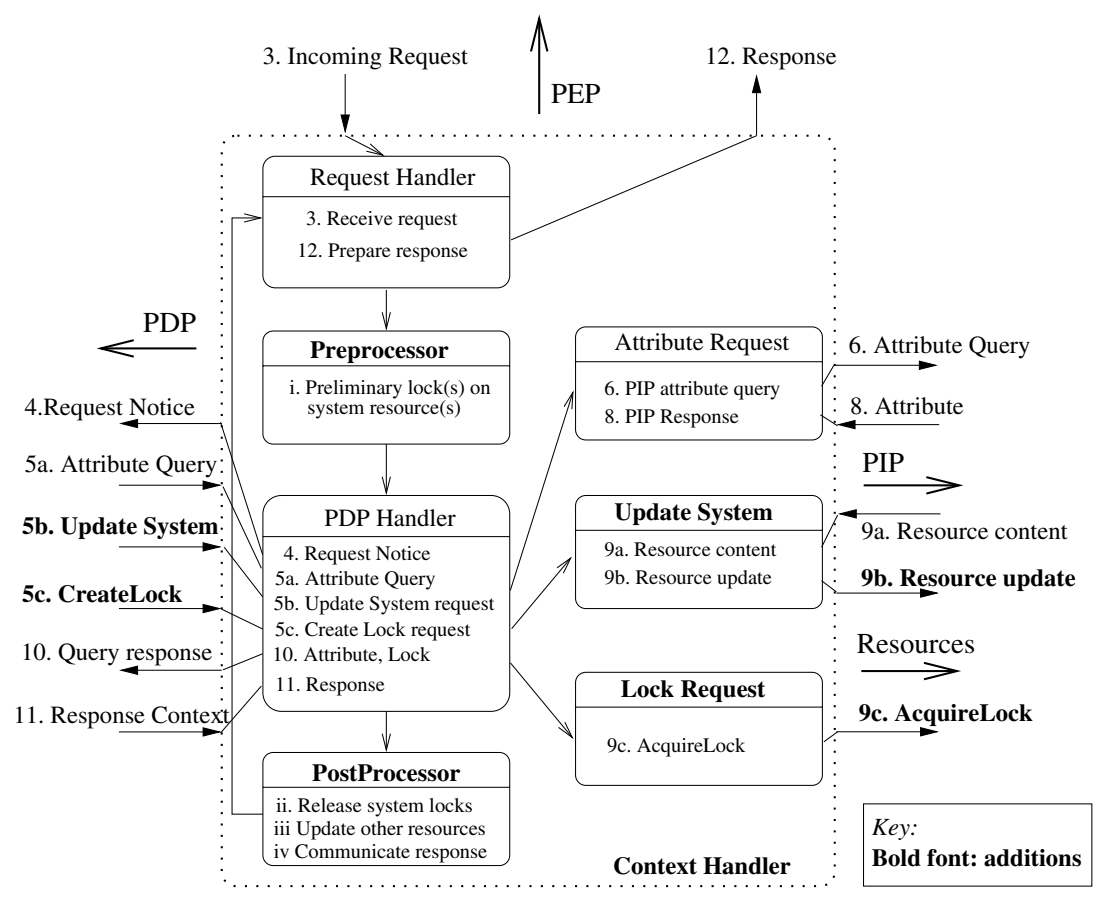

Fig. 3. Context Handler

Releasing a Lock. Lock release is an atomic process implemented as the method releaseLock, implemented atomically.

Verifying a Lock. The verifyLock operation verifies the validity of a lock. The execution model states that for every resource update must be preceded by a call to verifyLock, and is invoked by the resource manager for verifying the validity of a lock.

The execution model is that a requester gets the lock and at the time of resource usage and presents the locking permission to the resource manager that in turn verifies the validity of the presenter's claim with the lock manager and or PDP.

Registering with a Single LM. Registering a resource with a single lock manager is a basic design requirement enforced by requiring an attribute certificate where each resource is bound to a single lock manager (by a local certificate authority) using an X.509 attribute certificate [11].

\subsection{Enhancing the Context Handler}

We enhance the context handler with resource pool bootstrapping, termination and maintenance, (i.e., performing singular registration, deregistration, etc.) and extending the business logic for additional functionality. 
Resource Pool Maintenance. We extend the context handler to support the lock manager bootstrapping and maintenance of the resource pool. Algorithms in this section are written in pseudo code, with ' $->$ ' symbol indicating a call to a sub-module within the context handler.

Securely Registering Resources. To prevent multiple concurrent registration requests being invoked by a resource, we introduce the secure registration of resource procedure. Secure registration begins with a registration request by a resource, accompanied with a verifiable attribute certificate containing the resource and the lock manager. This request is serviced by Algorithm 1 below, as shown in figure 3

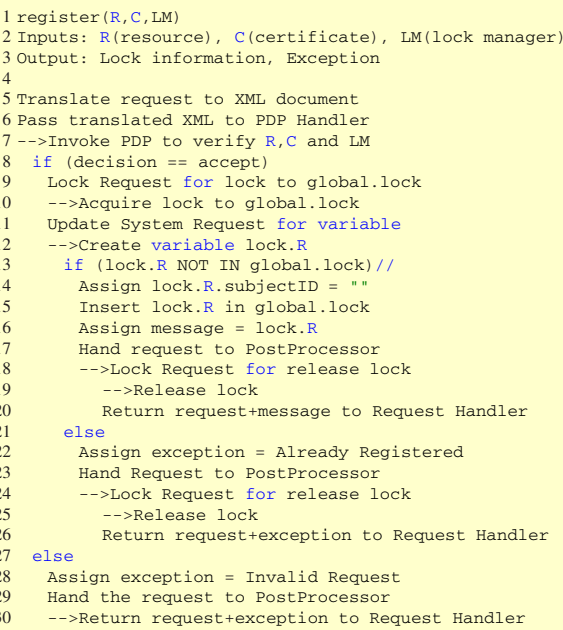

\section{Algorithm 1. Secure Registration}

The register method accepts three inputs $-\mathrm{R}$, the registering resource; $\mathrm{C}$, its attribute certificate, and LM, the Lock Manager. This request is handed to the 'Request handler' module of $\mathrm{CH}$ by the PEP (line 5). The request handler translates the request into XML and hands it to PDP handler for further processing (line 6). The PDP handler invokes the PDP and processes the response (lines 7,8). If the decision is accept, a lock is acquired (lines 9,10) and an update request is fired (line 11). Based on the success of this call, lock is release and the relevant message is returned to the request handler (lines 18-26). If PDP denies the register request, an appropriate response is constructed as well (line 27-30).

Securely Deregistering a Resource. Similar to registration, deregistration requires locking support because in order to prevent being deregistered while in use. The process flow is as follows: 


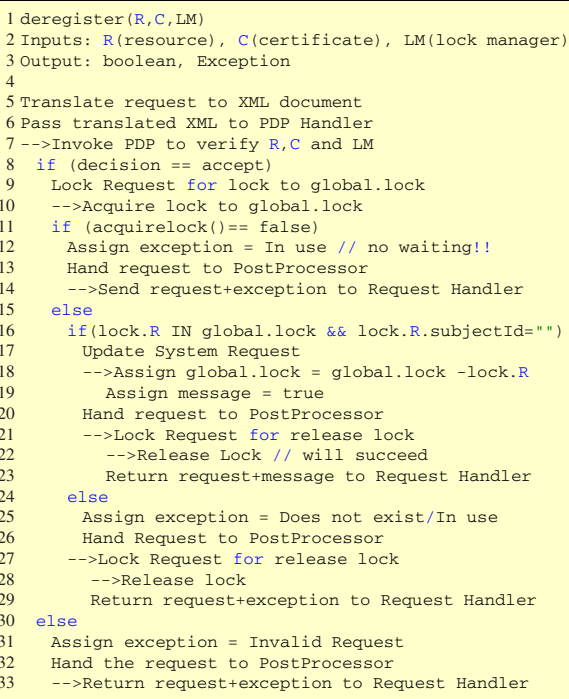

\section{Algorithm 2. Secure Deregistration}

Similar to the register method deregister accepts the same three inputs and securely removes the resource from LM. The difference here is that the update request removes the lock from a global lock data structure in a critical section (line 18).

\section{Extending the Business Logic}

Gaining Exclusive Access to Resources. Once a resource is registered, exclusive access to it can be guaranteed by a process very similar to the above processes, as follows:

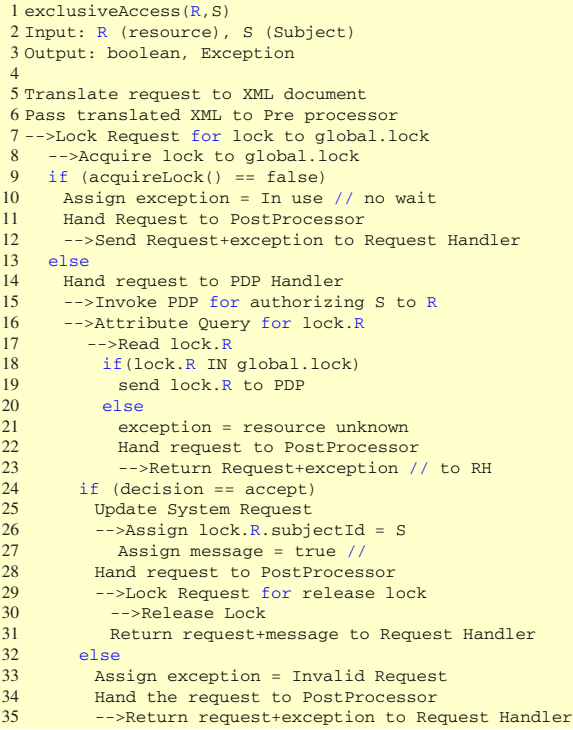


The exclusiveAccess method invokes a similar $\mathrm{CH}$ work-flow for granting access to externally usable resources. The main difference here is a call to the pre processor module that acquires locks before beginning any PDP evaluation (lines 7-12). In addition, the PDP may query for lock attributes (lines 16-23). Finally, if the request is granted, the lock manager updates lock. R to indicate the new owner (line 26).

Using Resource. Exercising exclusive access, once a requester has gained such an access to a resource, is a call to the resource (through the PEP). It is the resource's responsibility to ensure that the requester owns a valid lock to it, done by a verifyLock call to the lock manager (details are omitted due to lack of space).

Guaranteeing Dynamic/ History-Based Access Constraints. Steps for enforcing dynamic constraints are as follows:

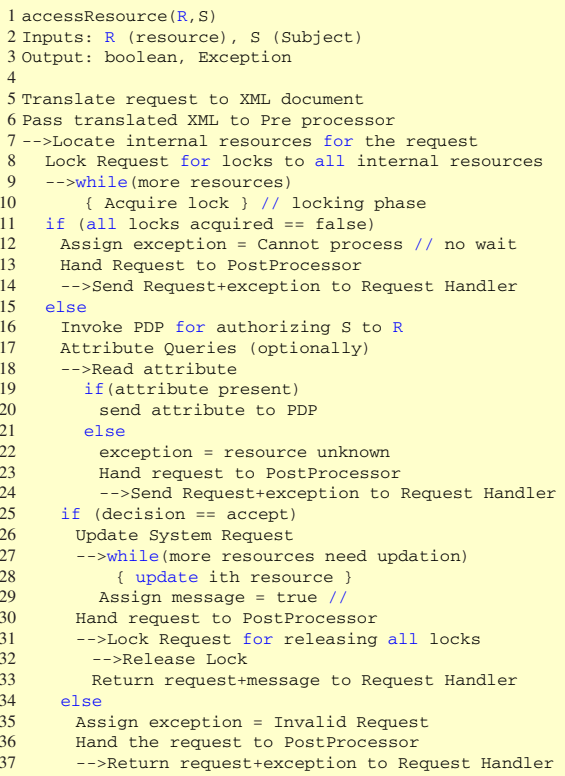

Algorithm 4. Dynamic constraints

Releasing Resource. A resource requester holding a lock can release the resource. A simple modification to the algorithms presented above does this, where the details are omitted due to lack of space.

\section{Implementing the Enhanced Design}

In this section presents the salient features of our LM implementation. We begin our discussion with data structures to implement locks, followed by a sample Java snippet and WSDL interaction to acquire locks. 


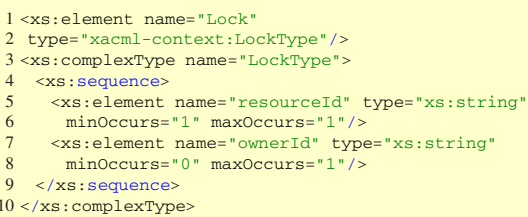

\section{Listing 1. The Lock Data Structure}

Listing 1 shows the LockType XML Schema (lines 3-10) that represents a lock for a single shared resource. The data structure identifies the lock through a resourceID and an ownerId. An available lock has an ownerId as an empty string, while a locked resource has a non-empty ownerId. Several such locks (one for each shared resource) are stored in a global LM-data structure called the GlobalLock.

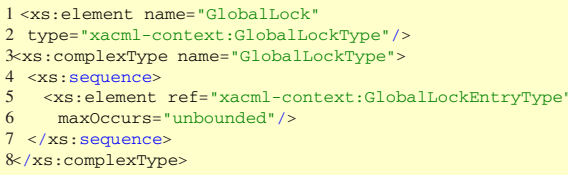

Listing 2. The Global Lock Data Structure

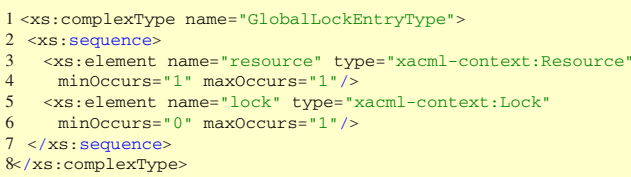

Listing 3. The Global Lock Entry Type

The Global Lock Entry Type data structure stores (key,value) pairs including resource and its locks.

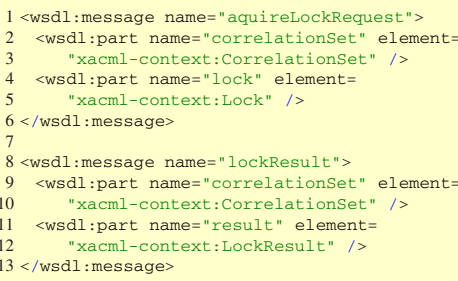

Listing 4. Lock acquisition/response message

Listing 3 shows WSDL message definitions for the acquireLock request and the result response. 


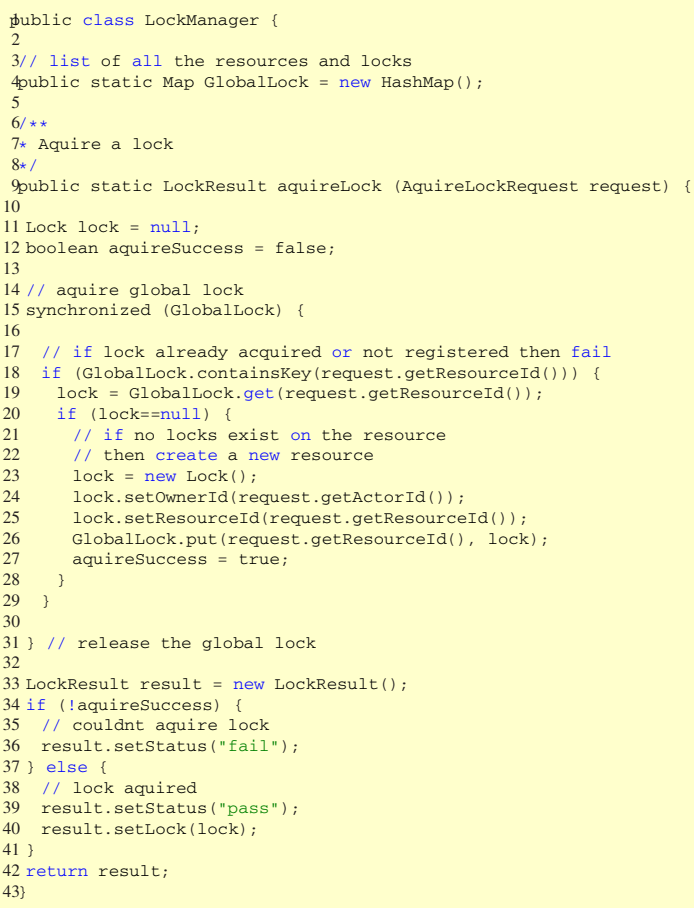

\section{Listing 5. AcquireLock method}

Listing 5 shows a Java implementation of the AcquireLock method for acquiring a lock to an existing resource. Java allows synchronization through exclusive access to objects that we leverage upon in this implementation (line 17). In Line 16 we aquire exclusive access to the GlobalLock data structure till Line 33. In line 19 we check if the resource is registered with the Lock Manager. Line 21: We check if the lock has already been granted, if not then we create a new Lock with the owner and resource specified in request and add it to GlobalLock (Line 24-27). Finally after updating the GlobalLock we remove our exclusive access to it (Line 33) Line 35: we create a result data structure. Line 36-43: we construct the result for the request accordingly and return it in line 44.

\section{Safety and Liveliness Properties}

Because we allow concurrent requests and use locks to serialize access to critical sections of the security monitor, we ensure liveliness and safety properties. In this section, we informally argue for them.

Lemma 1. Given a resource pool $\mathcal{R}$ and a set of lock managers $\mathcal{L}$, a resource $R_{i} \in \mathcal{R}$ can only be registered with a single lock manager $L_{j} \in \mathcal{L}$

Proof Sketch: See [10] for proof. 
Lemma 2. Given a resource $R_{i}$ and a lock manager $L_{K}$ to which $R_{i}$ can register itself, register method ensures that $R_{i}$ can be registered only once with $L_{K}$.

Proof Sketch: See [10] for proof.

Lemma 3. Given a resource $R_{i}$ and a lock manager $L_{K}$ to which $R_{i}$ is registered, deregister method ensures that only $R_{i}$ can be deregister itself from $L_{K}$.

Proof Sketch: See [10] for proof.

Theorem 1 (Safety of the exclusive access:). Given an XACML policy $P$ for exclusive access to an available resource $R$ and multiple concurrent access requests from subjects $S_{i}, i \in[1, n]$ (i.e., exclusiveAccess $\left(R, S_{i}\right)$ ), only one request from the above set is authorized by $P$.

Proof: See [10] for proof.

Theorem 2 (Safety of dynamic constraints:). Given an XACML policy $P$ for $d y$ namic constraint for access to a resource and multiple conflicting access requests (accessResource $\left.\left(R, S_{i}\right)\right)$, then $P$ authorizes only one request.

Proof: Similar to that of Theorem 1.

Theorem 3 (Liveliness1:). Given a resource $R$ and an exclusive use access request by subject $S$ exclusiveAccess $(\mathrm{R}, \mathrm{S}))$, then policy evaluation will release locks to all internal resources irrespective of the access control decision.

Proof Sketch: See [10] for proof.

Theorem 4 (Liveliness2:). Given resources $x, y$ and an exclusive use access requests by subject $S$ exclusiveAccess $(\mathrm{x}, \mathrm{S})$ followed by exclusiveAccess $(\mathrm{Y}, \mathrm{S})$ ) and concurrent exclusive use access requests by subject $T$ exclusiveAccess ( $\mathrm{y}, \mathrm{T})$ followed by exclusiveAccess $(\mathrm{x}, \mathrm{T})$ ), then at-least one of the exclusive access requests is denied by policy evaluation and all locks acquired for that policy evaluation are released.

Proof Sketch: See [10] for proof.

\section{Conclusions}

XACML is the default access control specification language for the World Wide Web [12 19]. But XACML does not currently support three types of access control Use Cases, viz., ensuring exclusive access to globally available resources, preventing access to a resource given a concurrent conflicting use of another resource (DSoD constraints), and preventing access to a resource given a history of conflicting access (such as in Chinese Wall constraints). We extend XACML syntax for supporting the abovementioned use cases, by enhancing the XACML policy enforcement framework with a lock manager, to realize the additional Use Cases, and informally argue that safety and liveliness properties are ensured by our implementation. 


\section{References}

1. Entrust:http://www.entrust.com/

2. Vordel:http://www.vordel.com/

3. Benatallah, B., Casasti, F., Toumani, F., Hamadi, R.: Conceptual modeling of web service conversations. Technical Report HPL-2003-60, HP Laboratories Palo Alto (March 2003)

4. Bhatti, R., Bertino, E., Ghafoor, A.: A trust-based context-aware access control model for web services. In: 2nd IEEE International Conference on Web Services (ICWS), July 2004, IEEE Computer Society Press, Los Alamitos (2004)

5. Bhatti, R., Joshi, J.B.D., Bertino, E., Ghafoor, A.: Access Control in Dynamic XML-Based Web Services using X-RBAC. In: First International Conference on Web Services ( ICWS) (June 2003)

6. Bhatti, R., Joshi, J.B.D., Bertino, E., Ghafoor, A.: X-GTRBAC Admin: A Decentralized Administration Model for Enterprise-Wide Access Control. In: 9th ACM Symposium on Access Control Models and Technologies (SACMAT), June 2005, ACM Press, New York (2005)

7. Bhatti, R., Joshi, J.B.D., Bertino, E., Ghafoor, A.: X-GTRBAC:An XML-Based Policy Specification Framework and Architecture for Enterprise-Wide Access Control. ACM Transactions on Information and System Security (TISSEC) 8(2) (2005)

8. Clark, D., Wilson, D.: A comparison of commercial and military computer security policies. In: IEEE Symposium on Security and Privacy, Oakland, April 1987, pp. 184-194. IEEE Computer Society Press, Los Alamitos (1987)

9. Clark, D., Wilson, D.: Evolution of a model for computer integrity. In: Eleventh National Computer Security Conference, Baltimore (October 1988)

10. Dhankhar, V., Kaushik, S., Wijesekera, D.: XACML policies for exclusive resource usage. Technical Report ISE-TR-07-03, ISE Department, George Mason University, Fairfax (April 2007)

11. Farrell, S., Housley, R.: RFC 3281- an internet attribute certificate (April 2002)

12. Ferraiolo, D.F., Sandhu, R., Gavrila, S., Kuhn, D.R., Chandramouli, R.: Proposed nist standard for role-based access control. ACM Transactions on Information and System Security 4(3), 224-274 (2001)

13. Haddad, S., Moreaux, P., Rampacek, S.: Client synthesis for Web Services by way of a timed semantics (ICEIS 06). In: 8th International Conference on Enterprise Information Systems (May 2006)

14. Joshi, J.B., Bertino, E., Latif, U., Ghafoor, A.: A generalized temporal role-based access control model. IEEE Transaction on Knowledge and Data Engineering 17(1) (Janurary 2005)

15. Lepro, R.: Cardea: Dynamic access control in distributed systems. Technical Report NAS03-020, NASA Advanced Supercomputing (NAS) Division, NASA Ames Research Center, Moffet Field, CA (November 2003)

16. OASIS: Business process execution language for web services (May 2003)

17. OASIS: Core and hierarchical role based access control (rbac) profile of xacml v2.0 (Feburary 2005), http://docs.oasis-open.org/xacml/2.0/access_controlxacml-2.0-rbac-profile1-specos.pdf

18. OASIS: Extensible access control markup language (Feburary 2005)

19. RFC 2753: A framework for policy-based admission control

20. Sandhu, R.S.: A lattice interpretation of the chinese wall policy. In: Proc. 15th NIST-NCSC National Computer Security Conference, pp. 329-339 (1992) 
21. Tanenbaum, A.S., Steen, M.v.: Distributed Systems: Principles and Paradigms. Prentice-Hall, Englewood Cliffs (2002)

22. Tannenbaum, A.S.: Modern operating systems. Prentice-Hall Inc., Englewood Cliffs, NJ (1992)

23. Tartanoglu, F., Issarny, V., Levy, N., Romanovsky, A.: Dependability in the web service architecture. In: ICSE Workshop on Architecting Dependable Systems, Orlando, FL (May 2002) 